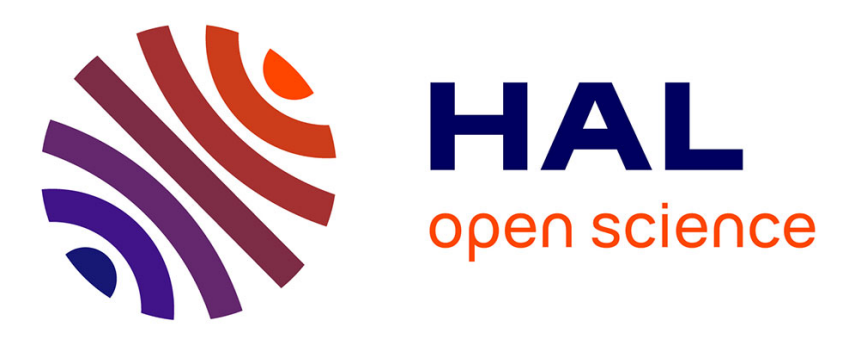

\title{
Hybrid Checkpointing for Parallel Applications in Cluster Federations
}

Sébastien Monnet, Christine Morin, Ramamurthy Badrinath

\section{To cite this version:}

Sébastien Monnet, Christine Morin, Ramamurthy Badrinath. Hybrid Checkpointing for Parallel Applications in Cluster Federations. 4th IEEE/ACM International Symposium on Cluster Computing and the Grid, Apr 2004, Chicago, IL, United States. inria-00000991v3

\section{HAL Id: inria-00000991 \\ https://hal.inria.fr/inria-00000991v3}

Submitted on 4 Mar 2016

HAL is a multi-disciplinary open access archive for the deposit and dissemination of scientific research documents, whether they are published or not. The documents may come from teaching and research institutions in France or abroad, or from public or private research centers.
L'archive ouverte pluridisciplinaire HAL, est destinée au dépôt et à la diffusion de documents scientifiques de niveau recherche, publiés ou non, émanant des établissements d'enseignement et de recherche français ou étrangers, des laboratoires publics ou privés. 


\title{
Hybrid Checkpointing for Parallel Applications in Cluster Federations
}

\author{
Sébastien Monnet \\ IRISA \\ Christine Morin \\ IRISA/INRIA \\ Sebastien.Monnet@irisa.fr \\ Christine.Morin@irisa.fr \\ Ramamurthy Badrinath \\ Hewlett-Packard ISO, Bangalore, India ${ }^{1}$ \\ badrinar@india.hp.com
}

\begin{abstract}
Cluster federations are attractive to execute applications like large scale code coupling. However, faults may appear frequently in such architectures. Thus, checkpointing longrunning applications is desirable to avoid to restart them form the beginning in the event of a node failure. To take into account the constraints of a cluster federation architecture, an hybrid checkpointing protocol is proposed. It uses global coordinated checkpointing inside clusters but only quasi-synchronous checkpointing techniques between clusters. The proposed protocol has been evaluated by simulation and fits well for applications that can be divided in modules with lots of communications within modules but few between them.
\end{abstract}

\section{Introduction}

We consider cluster federations. This kind of architecture may be used when some modules of a parallel application are running on different clusters. This can be the consequence of security rules (a module of an application may need to run into its owner laboratory), of hardware constraints (a module may use sensors or specific hardware to display results), or of large scale needs. An example of a code coupling application running in a cluster federation is different parallel simulation modules that sometimes need to communicate with each other.

There are lots of papers describing checkpoint / restart protocols inside a cluster in the literature. We want to take advantage of the high performance network (SAN) in the clusters and to take into account inter-cluster links which can be LANs or WANs for efficiently storing code coupling applications checkpoints. Considering the characteristics

\footnotetext{
${ }^{1}$ This work was done when R. Badrinath was a visiting researcher at IRISA, on leave from IIT Kharagpur
}

of a cluster federation architecture, different checkpointing mechanisms should be used within and between clusters. We propose an hybrid checkpointing protocol: it uses coordinated checkpointing within clusters and communicationinduced checkpointing between them.

Simulation of the protocol shows that it works well for code coupling applications.

The remainder of this paper is organized as follows. Section 2 presents the protocol design principles. Section 3 describes the proposed hybrid protocol combining coordinated and communication-induced checkpointing (called $\mathrm{HC}^{3} \mathrm{I}$ checkpointing protocol thereafter). Section 4 presents some of the algorithms. Section 5 is devoted to the evaluation of the protocol. In Section 6, related work is reviewed. Section 7 concludes.

\section{Design Principles}

This section presents the model considered in our work and the design principles of the $\mathrm{HC}^{3} \mathrm{I}$ checkpointing protocol.

\subsection{Model}

Application. We consider parallel applications like code coupling. Processes of this kind of application can be divided into groups (modules). Processes inside a same group communicate a lot while communications between processes belonging to different groups are limited. Communications may be pipelined as in Figure 1 or they may consist of exchanges between two simulation modules for example.

Architecture. We assume a cluster federation as a set of clusters interconnected by a Wide Area Network (WAN), inter-cluster links being either dedicated or even Internet, or a Local Area Network (LAN). Such an architecture is 


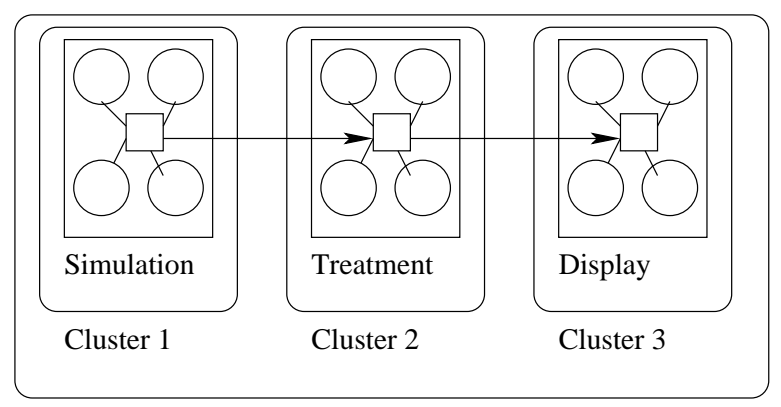

Cluster Federation

Figure 1. Application Model

suitable for the code coupling application model described above. Each group of processes may run in a cluster where network links have small latencies and large bandwidths (eg. System Area Networks (SAN)). We assume that a sent message will be received in an arbitrary but finite laps of time. This means that the network does not lose messages. This assumption implies that fault tolerance mechanisms should take care of in-transit messages, that should not be lost.

Failure assumptions. We assume that only one fault occurs at a time. However, the protocol can be extended to tolerate simultaneous faults as explained in Section ??. The failure model is fail-stop. It means that when a node fails it does not send messages anymore. The protocol takes into account neither omission nor byzantine faults.

\subsection{Checkpointing large scale applications in clus- ter federations}

The basic principle of all checkpoint / rollback methods is to periodically store an application consistent state to be able to restart from there in the event of a failure. A parallel application state is composed by the set of the states of all its processes. Consistent means that there is neither intransit messages (sent but not received) nor ghost messages (received but not sent) in the set of process states stored.

A message generates a dependency. For example, Figure 2 presents the execution of two processes which both store their local state ( $S 1$ and $S 2$ ). A message $m$ is sent from process 1 to process 2 . If the execution is restarted from the set of states $S 1 / S 2$ the message $m$ will have been received by process 2 but not sent by process 1 (ghost message). Process 1 will send $m$ again which is not consistent because $S 1$ happens before $S 2$. [4] defines the happen before relation with 3 rules: in a single process events are totally ordered; the emission of a message happens before its reception; the happen before relation is transitive. No happen before rela- tion should exist in the set of local states composing a global consistent state.

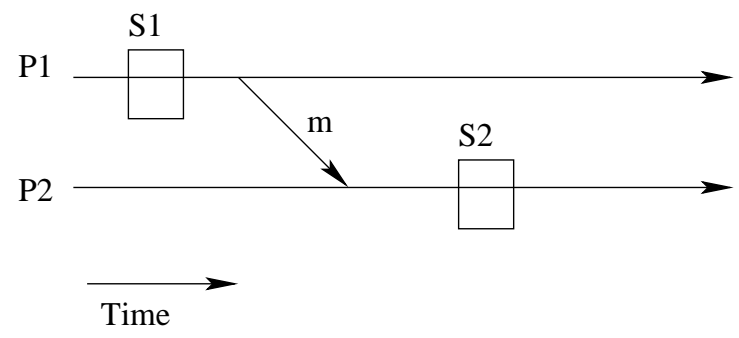

Figure 2. Dependency between two states

The last recorded consistent state is called the recovery line. [6] provides detailed information about the different checkpointing methods.

Inside a cluster we use a coordinated checkpointing method. This means there is a two-phase commit protocol during which application messages are frozen. It ensures that the stored state (the cluster checkpoint) is consistent. Coordinated checkpointing is affordable inside a cluster thanks to the high performance network (low latency and large bandwidth). Such techniques have already been implemented [8], [5],[11],[1].

The large number of nodes and network performance between clusters do not allow a global synchronization at the federation level. An independent checkpointing mechanism in which each cluster takes its Cluster Level Checkpoints (called CLC thereafter) does not fit. Tracking dependencies to compute the recovery line would be very hard at rollback time and clusters may rollback to very old CLCs (domino effect).

If we intend to $\log$ inter-cluster communications (to avoid dependencies), we need the piecewise deterministic $(P W D)$ assumption. The $P W D$ assumption means that we are able to replay a parallel execution in a cluster that produces exactly the same messages as the first execution. This assumption is very strong. Replaying a parallel execution means detecting, logging and replaying all nondeterministic events. It is not always possible.

The assumption that inter-cluster communications are limited leads us to use a communication-induced method between clusters. This means that each cluster takes $C L C$ independently, but information is added to each inter-cluster communication. It may lead the receiver of a message to take a $C L C$ (called forced $C L C$ ) to ensure the recovery line progress. Communication-induced checkpointing seems to keep enough synchronization and can be efficient.

So, we propose an hybrid protocol combining coordinated and communication-induced checkpointing $\left(\mathrm{HC}^{3} \mathrm{I}\right)$. 


\section{Description of the $\mathrm{HC}^{3} \mathrm{I}$ Checkpointing Protocol}

In this section we first present the checkpointing mechanism used in a cluster. We then describe mechanisms used to track inter-cluster dependencies and to decide when a $C L C$ should be forced. Finally, we describe the rollback protocol and the garbage collector needed to eliminate $C L C$ that are no longer useful.

\subsection{Cluster level checkpointing}

In each cluster, a traditional two-phase commit protocol is used. An initiator node broadcasts (in its cluster) a CLC request (see Algorithm 3 in Section 4.4). All the cluster nodes acknowledge the request, then the initiator node broadcasts a commit. Our implementation of the two-phase commit protocol is described in Algorithm 4 in Section 4.4. Between the request and the commit messages, application messages are queued to prevent intra-cluster dependencies (see Algorithm 5 in Section 4.5).

In order to be able to retrieve $C L C$ data in the event of a node failure, $C L C s$ are recorded in the node own memory, and in the memory of one other node in the cluster. Because of this stable storage implementation, only one simultaneous fault in a cluster is tolerated.

Each $C L C$ is numbered. Each node in a cluster maintains a sequence number $(S N)$. $S N$ is incremented each time a $C L C$ is committed. This ensures that the sequence number is the same on all the nodes of a cluster (outside the twophase commit protocol). The $S N$ is used for inter-cluster dependency tracking. Indeed, each cluster takes its $C L C$ periodically, independently from the others.

\subsection{Federation level checkpointing}

If we look at our application model, communications between two processes in different clusters may appear, which imply dependencies between $C L C s$ taken in different clusters. Dependencies need to be tracked to be able to restart the application from a consistent state.

Forcing a $C L C$ in the receiver's cluster for each inter-cluster application message would work but the overhead would be huge as it would force useless checkpoints. In Figure 3, cluster 2 takes two forced CLCs (the filled ones) at message reception, and the application takes received message into account only when the forced $C L C$ is committed. CLC2 is useful: in the event of a failure, a rollback to $C L C 1 / C L C 2$ is consistent ( $m l$ would be sent and received again). On the other hand, forcing $C L C 3$ is useless: cluster 1 has not stored any $C L C$ between its two message sending operations. In the event of a failure it will have to rollback to $C L C 1$ which will force cluster 2 to rollback to $C L C 2$. CLC3 would have been useful only if cluster 1 would have stored a $C L C$ after sending $m l$ and before sending $m 2$.

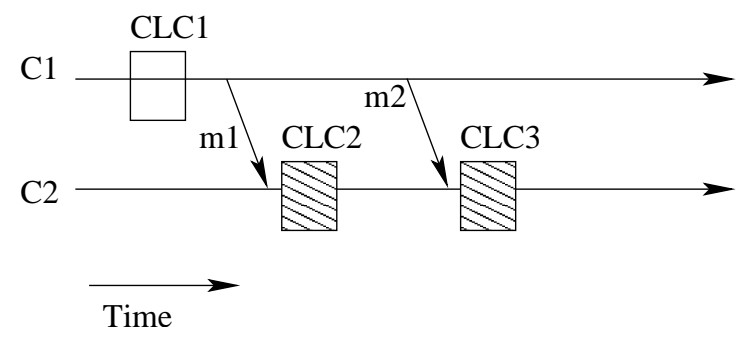

Figure 3. Limitation of the number of forced CLCs

Thus, a $C L C$ is forced in the receiver's cluster only when a $C L C$ has been stored in the sender's cluster since the last communication from the sender's cluster to the receiver's cluster. To this end, CLCs are numbered in each cluster with a $S N$ (as described in previous section). The current cluster's sequence number is piggy-backed on each intercluster application message (Section 4.1 describes the message data structure). To be able to decide if a $C L C$ needs to be initiated, all the processes in each cluster need to keep the last received sequence number from each other cluster. All these sequence numbers are stored in a $D D V$ (Direct Dependencies Vector, [2]). How the receiver decides if it needs to initiate a forced $C L C$ is shown in Algorithm 6 in Section 4.5.

$D D V_{j}[i]$ is the $\mathrm{i}^{\text {th }} D D V$ entry of cluster $j$, and $S N_{i}$ is the sequence number of cluster $i$.

For a cluster $j$ :

If $i=j, D D V_{j}[i]=S N_{j}$

If $i \neq j, D D V_{j}[i]=$ last received $S N_{i}$ (0 if none).

Note that the size of the $D D V$ is the number of clusters in the federation, not the number of nodes. In order to have the same $D D V$ and $S N$ on each node inside a cluster, we use the synchronization induced by the $C L C$ two-phase commit protocol to synchronize them (as described in Algorithm 4 in Section 4.4). Each time the $D D V$ is updated, a forced $C L C$ is initiated which ensures that all the nodes in the cluster which take a $C L C$ will have the same $D D V$ at commit time. The current $D D V$ is stored with each $C L C$.

\subsection{Logs to avoid huge rollbacks}

Coordinated checkpointing implies to rollback the entire cluster of a faulty node. We want to limit the number of clusters that rollback. If the sender of a message does not rollback while the receiver does, the sender's cluster does not need to be forced to rollback. When a message is sent outside a cluster, the sender logs it optimistically in its volatile memory (logged messages are used only if the sender does not rollback). This is shown by Algorithm 5 . 
The message is acknowledged with the receiver's $S N$ which is logged along with the message itself (Algorithm 7). Next section explains which messages are replayed in the event of a failure.

\subsection{Rollback}

If a node fails inside a cluster, it is detected and the cluster rolls back to its last stored $C L C$ (the description of the failure detector is out of the scope of this paper). One node in each other cluster of the federation receives a rollback alert. It contains the faulty cluster's $S N$ that corresponds to the $C L C$ to which it rolls back.

When a node receives such a rollback alert from another cluster with its new $S N$, it checks if its cluster needs to rollback by comparing its $D D V$ entry corresponding to the faulty cluster to the received $S N$. If the former is greater than or equal to the latter its cluster needs to rollback to the first (the older) $C L C$ which has its $D D V$ entry corresponding to the faulty cluster greater than or equal to the received $S N$. The node that has received the alert initiates the rollback. If a cluster needs to rollback due to a received alert, it will send a rollback alert containing its new $S N$ to alert all the other clusters. This is how the recovery line is computed.

Even if its cluster does not need to rollback a node receiving a rollback alert broadcasts it in its cluster. The nodes which have logged messages sent to a node in the faulty cluster and acknowledged with a $S N$ greater than the alert one or not acknowledged at all, re-send them.

Our communication-induced mechanism implies that clusters need to keep multiple $C L C$ and logged messages. They need to be garbage collected.

\subsection{Garbage collection}

Our protocol needs to store multiple $C L C s$ in each cluster in order to compute the recovery line at rollback time. The memory cost may become important. Periodically, or when a node memory saturates, a garbage collection is initiated. The garbage collector algorithm is centralized. A node initiates a garbage collection, it asks one node in each cluster to send back its list of all the $D D V s$ associated with the stored CLCs. Then it simulates a failure in each cluster and keeps for each ones the worst $S N$ to which they might rollback. It sends a vector containing all the worst $S N s$ to one node in each cluster which broadcasts it in its cluster. Each node removes the CLCs which have its cluster $D D V$ entry smaller than the worst $S N$ associated to its cluster. They also remove loggeqd messages that are acknowledged with a $S N$ smaller than the receiver's cluster worst $S N$.

\section{Algorithms}

This section presents the main algorithms of the $\mathrm{HC}^{3} \mathrm{I}$ protocol. More details can be found in [7] (in French). To make it simple we introduce the notion of leader. In each cluster one primary leader and one secondary leader are chosen (in a static way at the initialization). These nodes are responsible for failure detection, restarting faulty nodes and inter-cluster protocol communications (rollback alert and garbage collection messages). The algorithms are not detailed, for example, takeTentativeC $\operatorname{PPt}()$ means storing the local state locally and on another node (and waiting for an acknowledgement).

Each cluster has a unique ID, and in each cluster, each node has also a unique rank. Algorithm 8 about garbage collection messages is given in Section 4.6.

\subsection{Data structures}

We first present data structures used in the algorithms.

Constants:

- nbClusters number of clusters.

- myClusterId $i$ the ID of cluster $i$.

- nbNodes $i$ the number of nodes in cluster $i$.

- myRank $k_{i, j}$ the ID of node $j$ in the cluster $i$.

- lSet $_{i}$ set of cluster $i$ leaders.

- otherLeaders $i$ set of the other clusters leaders - in each cluster, the leaders have to be able to communicate with the others.

Timers:

- iMALIVETimer delay between heartbeats for the failure detection.

- chCkAliveTimer delay during which we should have receive at least one heartbeat from every node in a cluster.

- tentativeCkPtTimer maximum time between a checkpoint request and its corresponding commit.

- waitForAllTimer maximum time to wait after a checkpoint request for receiving all acknowledgments.

- gCTimer time between garbage collections.

- ckPtTimer time between two unforced CLCS.

Others:

- $m y S n_{i, j}$ the sequence number.

- $m y D D V_{i, j}$ the $D D V$.

- duringCkPt $t_{i, j}$ a boolean to know if a node is currently in the two-phase commit protocol (i.e. checkpointing).

- $h b_{i, j}$ a vector with $n b N o d e s_{i}$ entries to remember the received heartbeats.

- oldHb $b_{i, j}$ a copy of $h b_{i, j}$.

- ckPtAckSet ${ }_{i, j}$ set of nodes that have acknowledge a checkpoint request. 
- gcAckSet $_{i, j}$ set of nodes that have acknowledge a garbage request.

- initiator $_{i, j}$ rank of the last $C L C$ initiator.

Logs Each node logs in volatile memory messages related to inter-cluster communications: the message itself, the receiver's ID, and the sequence number of the receiver (known by the message acknowledgement).

\subsection{Initialization}

Algorithm 1 is the initialization sequence, it is executed by each node in the cluster federation at launch time. It sets the $D D V$, the sequence number, some variables and initializes some timers.

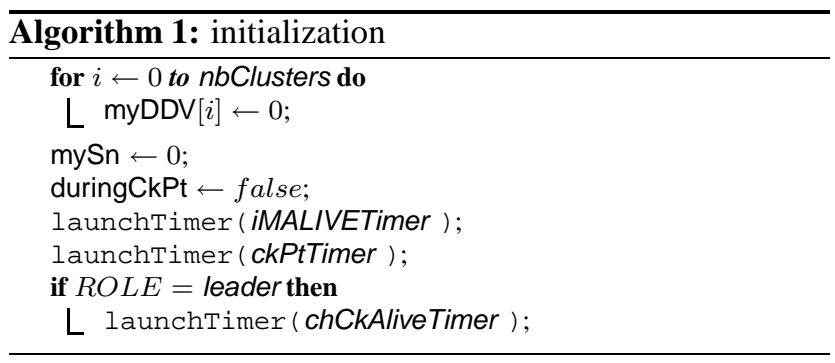

\subsection{Messages structure}

Messages exchanged by nodes have the following structure:

- sender the identity of the sender (sender.rank and sender.clusterId

- type (see Message dispatching algorithm).

- subtype (see ckPtHandler algorithm).

- $s n$ the sender's sequence number.

- data the message itself.

In Section 3.2, it is explained why messages need to contain $s n$, for dependencies tracking. It is used by the receiver to know if it needs to take a forced CLC. Algorithm 2 dispatches a message according to its type and its sender.

\subsection{Checkpointing algorithms}

Algorithm 3 initiates a $C L C$ in a cluster, as it is explained in Section 3.1.

Algorithm 4 is executed when checkpointing messages are received. It describes the implementation of the twophase commit protocol introduced in Section 3.1.

The names of the functions are used to describe what they do. For example, launchTimer launches a timer, and sendCkPtAck(id,sn) sends an acknowledgment (i.e. the type of the message is CKPT and its subtype is ACK) with $s n$ to the
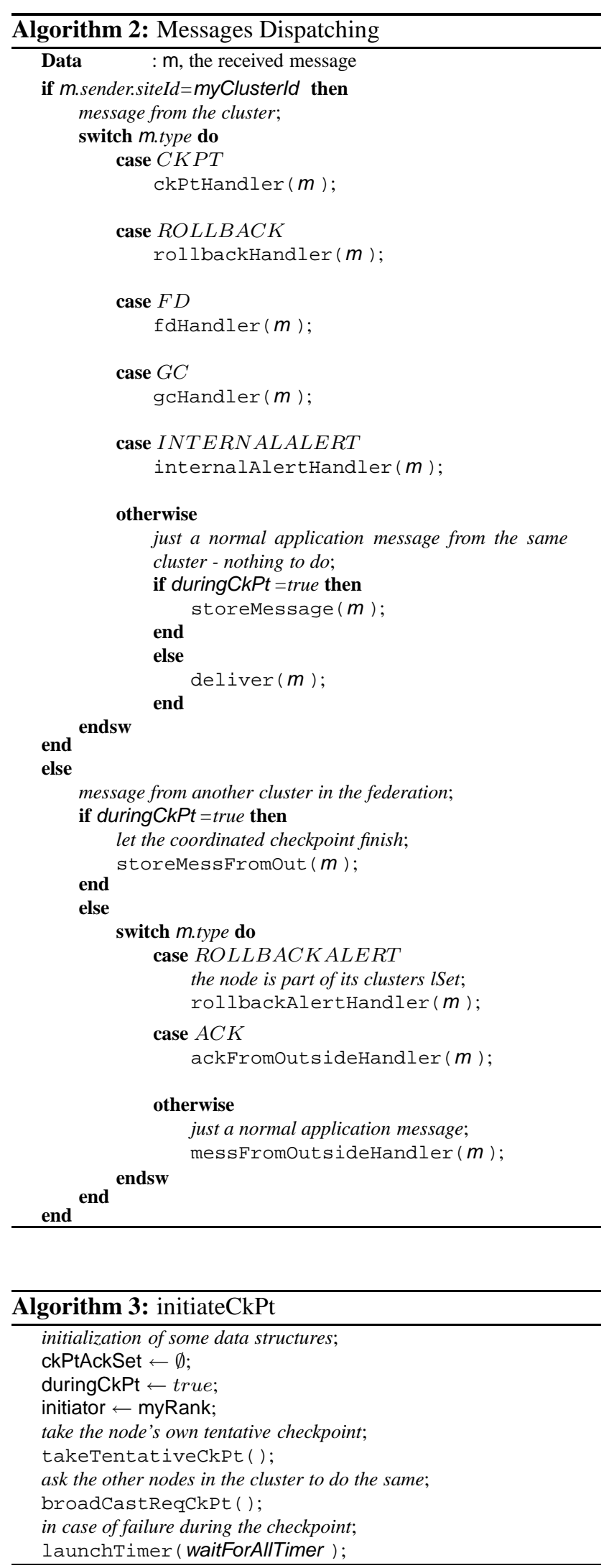
node represented by $i d$.

We can see that the synchronization induced by the twophase commit protocol also synchronizes the $D D V$ on all the cluster nodes.

\subsection{Application messages transmission}

Algorithm 5 is executed when a process is sending a message to another one in the cluster federation. The message is caught by the fault-tolerance layer, which puts the right message type and subtype, the sender identity and the current value of the sequence number. The fault-tolerance layer also checks if the message needs to be queued (if communications are frozen due to a checkpoint currently being stored) or logged (if it is an inter-cluster message).

Algorithm 6 is called when a message is received from an other cluster. It checks if a $C L C$ has to be initiated by comparing the received sequence number and its corresponding $D D V$ entry as explained in Section 3.2. Messages are acknowledged with the appropriate sequence number.

Algorithm 7 represents the fact that inter-cluster messages are logged (by the sender) with the sequence number that the receiver has at reception time, as soon as they are acknowledged.

\subsection{Garbage collection}

Algorithm 8 draws what is done for garbage collection. As described in Section 3.5, initiating a garbage collection means sending a request for garbage collection to all the leaders in the federation. Then, this algorithm shows what a node does when receiving such a request (it sends its entire $D D V$ list, one $D D V$ per $C L C$ stored). When all the $D D V$ lists have been received by the initiator, it computes the recovery line (explained in Section 3.5) then sends it to all other leaders in the cluster federation. If a leader receives such a message it broadcasts it in its cluster and every node collects all its obsolete data.

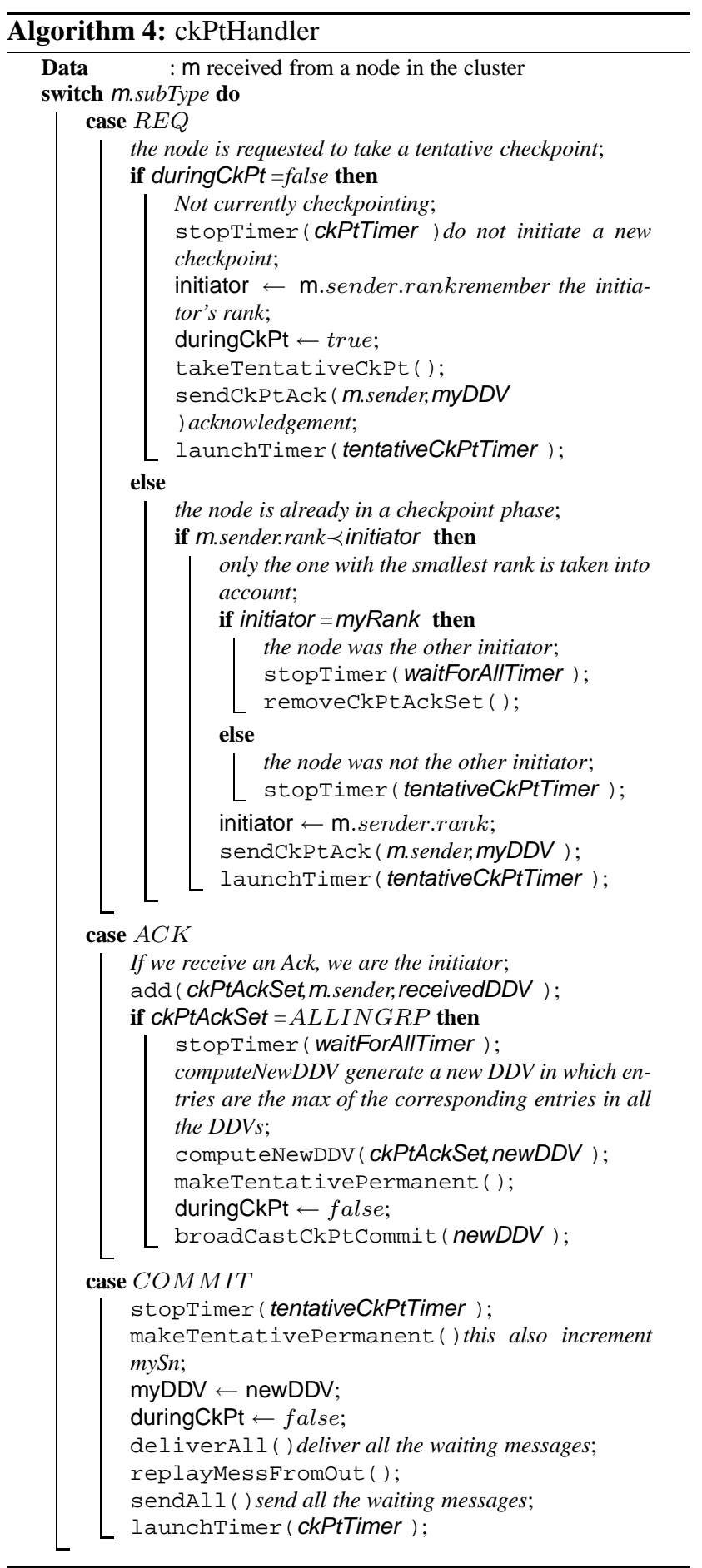



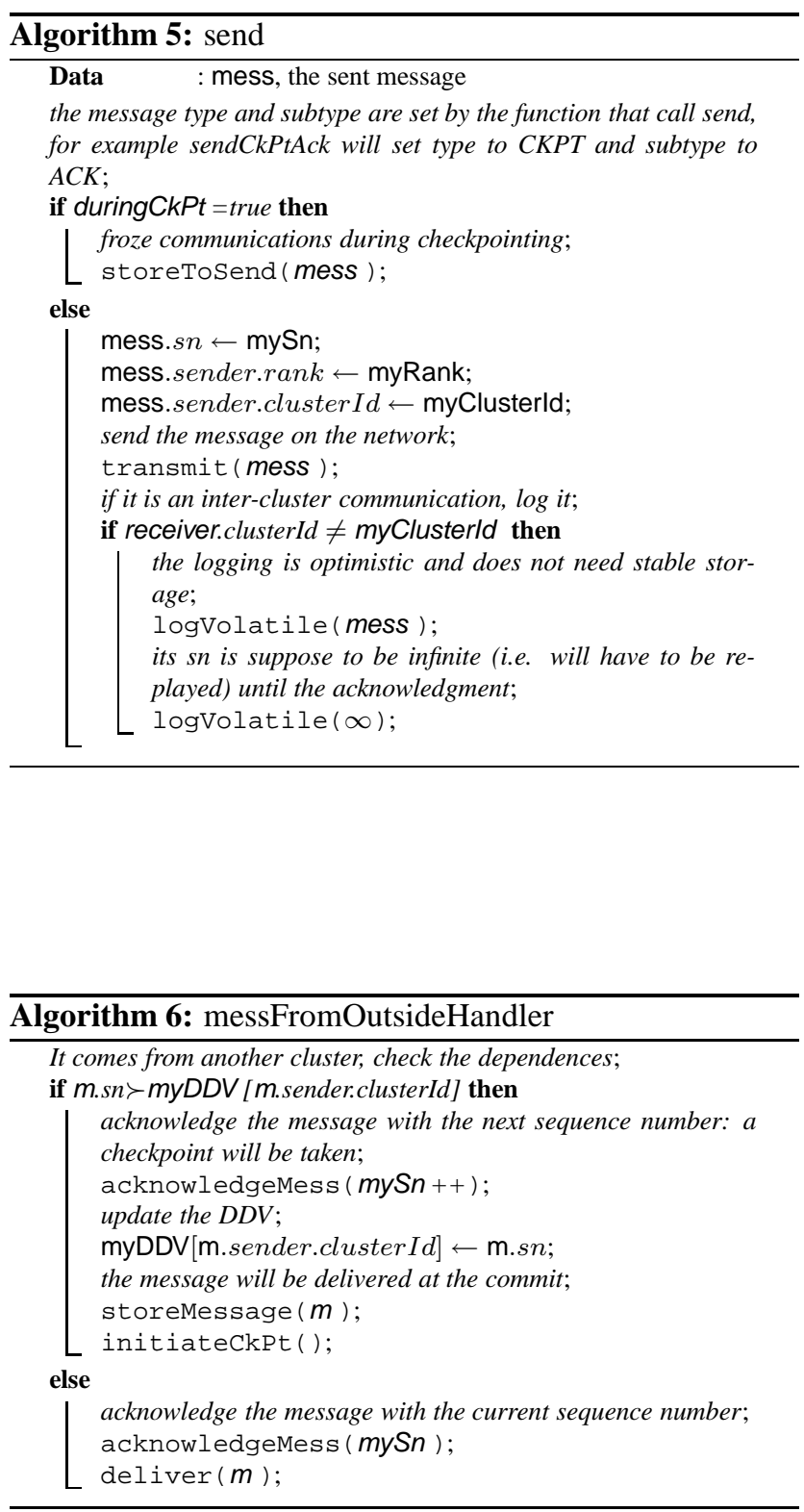

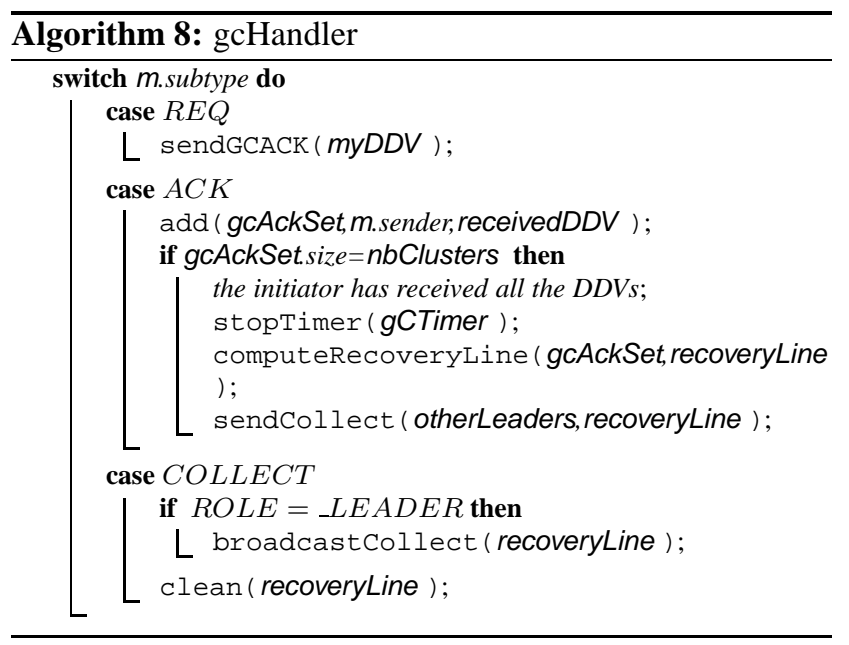

\section{Evaluation}

To evaluate the protocol, a discrete event simulator has been implemented. We have evaluated the overhead of the protocol in terms of network and storage cost first, then we observe what happens with different communication patterns. At last the garbage collector effectiveness and cost are evaluated.

\subsection{Simulator}

C++SIM library [12] has been used to write the simulator. This library provides generic threads, a scheduler, random flows and classes for statistical analysis. Our simulator is configurable. The user has to provide three files: a topology file, an application file and a timer file. In the topology file, there are the number of clusters, the number of nodes in each cluster, the bandwidth and latency in each cluster and between clusters (represented as a triangular matrix) and the federation MTBF (Mean Time Between Failures). The application file contains, for each cluster, the nodes mean computation times, communication patterns between computations (represented by send probabilities between nodes) and the application total time. At last, the timers file contains the delays for the protocol timers for each cluster (delays between two CLCs, garbage collection,...).

The simulator is composed of four main threads. The thread Nodes takes the identity of all the nodes, one by one. The thread Network stores the messages and computes their arrival time. The thread Timers simulates all the different timers. The thread Controller controls the other threads (launches them, displays results at the end,...). Communication between threads is performed by shared variables. The simulator can be compiled with different trace levels. In the higher, we can observe each node action time-stamped (sends, receives, timer interruptions, log searches...). The lowest simulator output is statistical data, as messages count 


\begin{tabular}{|c|c|c|}
\hline $\begin{array}{c}\text { Sender's } \\
\text { Cluster }\end{array}$ & $\begin{array}{c}\text { Receiver's } \\
\text { Cluster }\end{array}$ & $\begin{array}{c}\text { Message } \\
\text { Count }\end{array}$ \\
\hline Cluster 0 & Cluster 0 & 2920 \\
\hline Cluster 1 & Cluster 1 & 2497 \\
\hline Cluster 0 & Cluster 1 & 145 \\
\hline Cluster 1 & Cluster 0 & 11 \\
\hline
\end{tabular}

Table 1. Application messages

in clusters and between clusters, number of stored $C L C s$, number of protocol messages,...

\subsection{Network traffic and storage cost}

Evaluating network traffic and storage cost is very hard. It depends on how the protocol is tuned. If the frequency of unforced $C L C S$ is low in a cluster, the $S N S$ will not grow too fast so inter-cluster messages from this cluster would have a low probability to force $C L C s$. Reducing the protocol overhead becomes easy. If no $C L C$ is initiated, the only protocol cost consists in logging optimistically in volatile memory inter-cluster messages and transmitting an integer $(S N)$ with them. There is also a little overhead due to message interception (between the network interface and the application).

To take advantage of the protocol, the timer that regulates the frequency of unforced $C L C s$ in a cluster should be set to a value that is much smaller than the MTBF of this cluster. To illustrate this, the simulator simulates 2 clusters of 100 nodes. In both clusters the network is Myrinet like ( $10 \mu$ s latency and $80 \mathrm{Mb} / \mathrm{sec}$ bandwidth). The clusters are linked by Ethernet like links $(150 \mu$ s latency and $100 \mathrm{Mb} / \mathrm{sec}$ bandwidth). The application total execution time is 10 hours. There are lots of communications inside each cluster and few between them. It can be a simulation running on cluster 0 and a trace processor on cluster 1 for example. Table 1 displays the number of intra and inter-cluster messages.

Graph 4 and 5 show the number of forced and unforced committed CLCs in each cluster according to the delay between unforced $C L C S$ in cluster 0 (x axis, in minutes). Cluster 1 delay between $C L C s$ is set to infinite. Cluster 0 stores some forced CLCs (8) because of the communications from cluster 1 . This number of forced $C L C s$ is constant - there are few messages from cluster 1 . Notice that the total number of stored CLCs is smaller than $\frac{\text { totalcomputationtime }}{\text { delaybetweenCLCs }}+$ number of forced CLCs because the timer is reset when a forced $C L C$ is established. Clusters store few more CLCs, but they are better placed (in time). Cluster 1 does not store any unforced $C L C s$ as its timer is set to infinite, but it stores some forced $C L C s$ induced by incoming communications

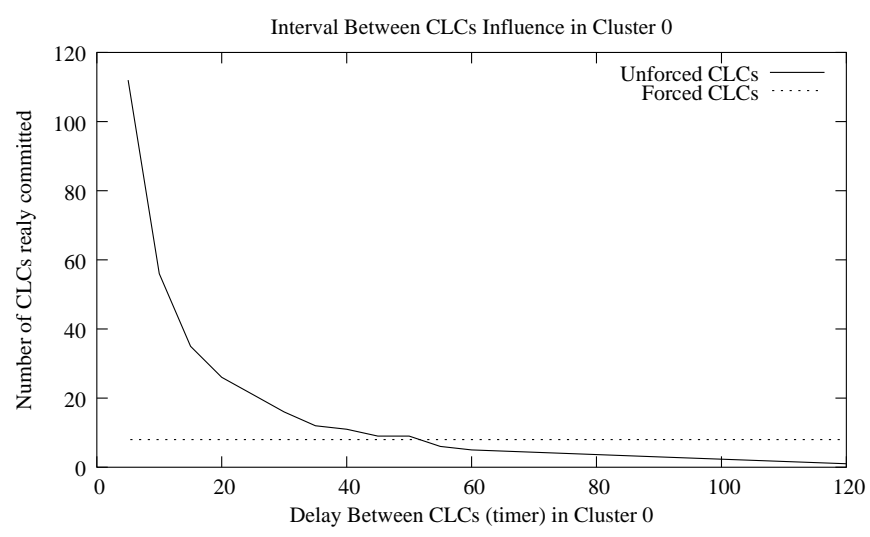

Figure 4. Number of CLCs in Cluster 0

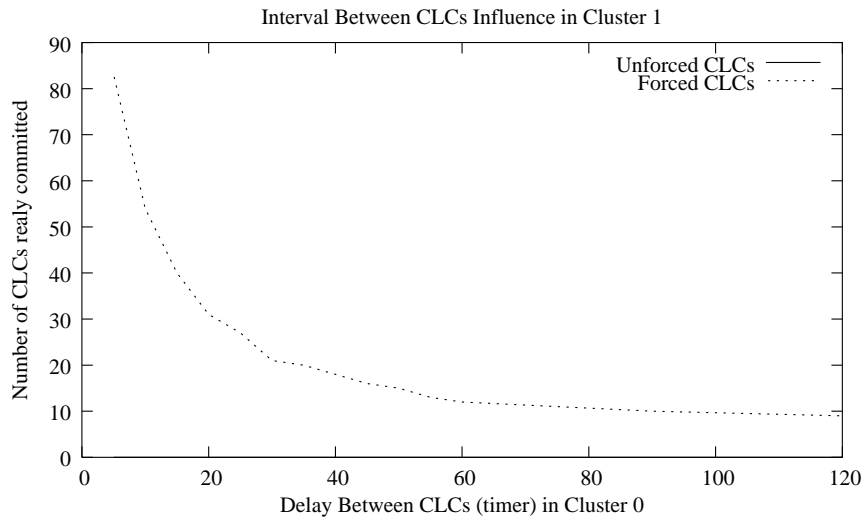

Figure 5. Number of CLCs in Cluster 1

from cluster 0 . The number of these forced $C L C s$ is proportional to the number of $C L C s$ stored in cluster 0 - numerous messages come from cluster 0 .

One may want to store more $C L C s$ in cluster 1 , if this cluster is intensively used and computation time is expensive for example. Graph 6 shows that cluster 0 (which "delay between $C L C s$ " timer is set to 30 minutes) does not store more $C L C s$ even if cluster 1 timer is set to 15 minutes. This is thanks to the low number of messages from cluster 1 to cluster 0 .

\subsection{Communication patterns}

To better understand the influence of the communications patterns on the checkpointing protocol, Graph 7 shows what happens when the number of messages from cluster 1 to cluster 0 increases. Both cluster "delay between $C L C s$ " timers are set to 30 minutes. The application is the same as in previous section except for the number of messages from cluster 1 to cluster 0 , which is represented on the $\mathrm{x}$ axis. 


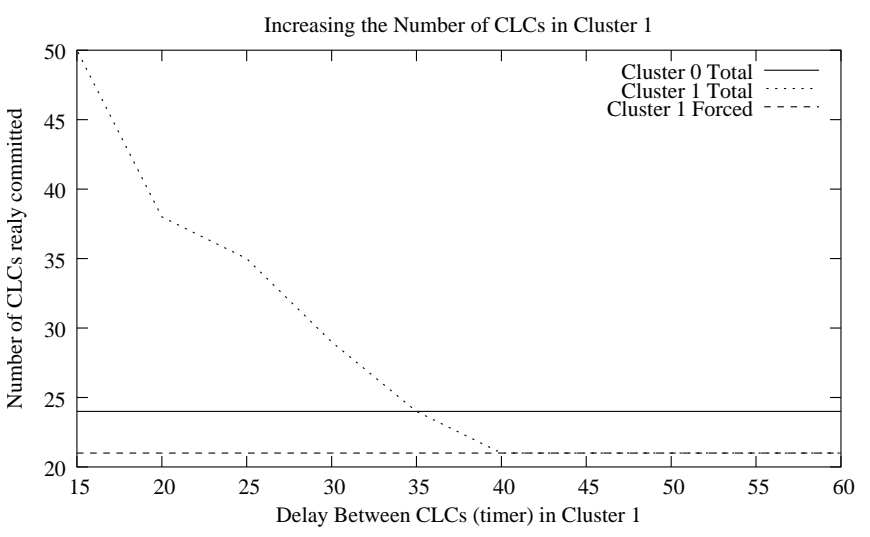

Figure 6. Impact of the Number of CLCs in Cluster 1

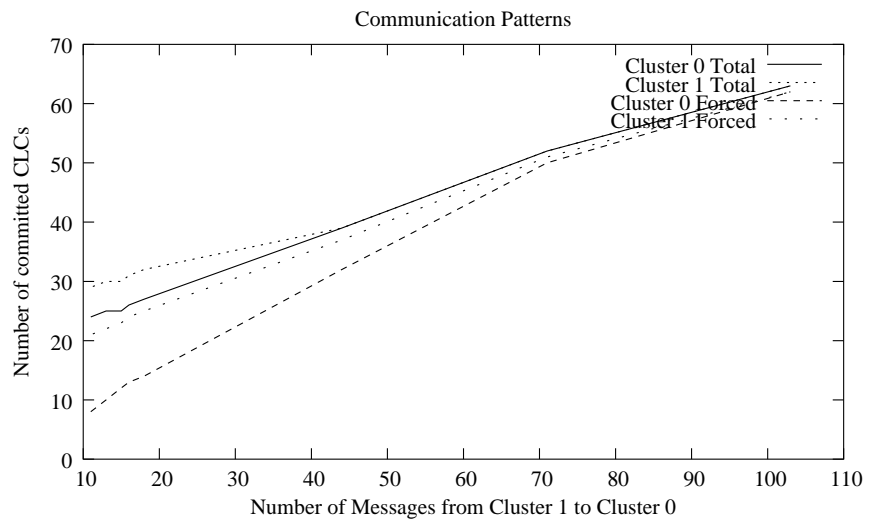

Figure 7. Increasing Communication from Cluster 1 to Cluster 0

The number of forced $C L C s$ increases fast with the number of messages from cluster 1 to cluster 0 . If the two clusters communicate a lot in both ways, $S N s$ grow very fast and most of the messages induce a forced $C L C$.

\subsection{Garbage collection}

The execution of a garbage collection may incur a non negligible overhead. If $N$ is the number of clusters in the federation, each garbage collection implies:

$N-1$ inter-cluster requests,

$\mathrm{N}-1$ inter-cluster responses which contains the list of all the $D D V s$ associated to the stored $C L C s$ in a cluster,

$N$-1 inter cluster collect requests,

A broadcast in each cluster.

However, our hybrid checkpointing protocol may store multiple $C L C s$ in each cluster. They can become very numer-

\begin{tabular}{|c|c|c|c|}
\hline $\begin{array}{c}\text { Cluster 0 } \\
\text { Before }\end{array}$ & $\begin{array}{c}\text { Cluster 0 } \\
\text { After }\end{array}$ & $\begin{array}{c}\text { Cluster 1 } \\
\text { Before }\end{array}$ & $\begin{array}{c}\text { Cluster 1 } \\
\text { After }\end{array}$ \\
\hline 10 & 2 & 11 & 2 \\
\hline 18 & 2 & 18 & 2 \\
\hline 15 & 2 & 14 & 2 \\
\hline 14 & 2 & 15 & 2 \\
\hline
\end{tabular}

Table 2. Number of stored CLCs (2 clusters)

\begin{tabular}{|c|c|c|c|c|}
\hline Cluster 0 (before) & 30 & 48 & 54 & 38 \\
\hline Cluster 0 (after) & 2 & 2 & 2 & 2 \\
\hline Cluster 1 (before) & 50 & 80 & 78 & 64 \\
\hline Cluster 1 (after) & 2 & 2 & 2 & 2 \\
\hline Cluster 2 (before) & 50 & 80 & 78 & 64 \\
\hline Cluster 2 (after) & 2 & 2 & 2 & 2 \\
\hline
\end{tabular}

Table 3. Number of stored CLCs (3 clusters)

ous. It also logs every inter-cluster application message. For the sample above, in the case of 103 messages sent from cluster 1 to cluster 0 , without any garbage collection, there are $63 C L C s$ in each cluster. It means that each node in the federation stores 126 local states (its own 63 local states and the ones of one of its neighbor, because of the stable storage implementation).

If a garbage collection is launched every 2 hours, the maximum number of stored $C L C s$ just after a garbage collection is 2 per cluster in this sample. Only oldest CLCs are removed, as explained in Section 3.5. So rollbacks will not be too deep. The maximum number of logged messages during the execution in the sample above is 4 in both clusters. Table 2 shows for each garbage collection the number of $C L C s$ stored just before and just after the collection. A second experimentation simulates an application that runs on three clusters. Clusters 0 and 1 have the same configuration as above. Cluster 2 is a clone of cluster 1 . There are approximately 200 messages that leave and arrive in each cluster. Table 3 shows for each garbage collection the number of CLCs stored just before and just after the collection.

A tradeoff has to be found between the garbage collection frequency and the number of $C L C s$ stored.

\section{Related work}

A lot of papers about checkpointing methods can be found in the literature. However, most of the previous works are related to clusters, or small scale architectures. A lot of systems are implemented at the application level, partitioning the application processes into steps. Our protocol is implemented at system level so that programmers do not need to write specific code. Moreover the protocol in this pa- 
per takes cluster federation architectures into account. This section presents several works that are close to ours.

Integrating fault-tolerance techniques in grid applications. [9] does not present a protocol for fault tolerance but it describes a framework that provides hooks to help developers to incorporate fault tolerance algorithms. They have implemented different well-known fault tolerance algorithms and it seems to fit well with large scale. However, these algorithms are implemented at application level and are made for object-based grid applications.

MPICH-V. [3] describes a fault tolerant implementation of MPI. It is made for large scale architectures. All the communications are logged and can be replayed. This avoids all dependencies so that a faulty node will rollback, but not the others. But this means that strong assumptions upon determinism have to be taken. Our protocol does not make any assumption on the application determinism. Moreover it takes advantage of the fast network available in the clusters.

Hierarchical coordinated checkpointing. The work presented in [10] is the closest from ours. It proposes a coordinated checkpointing method, based on the two-phase commit protocol. The synchronization between two clusters (linked by slower links) is relaxed. In [10], it is the coordinated checkpointing mechanism that is relaxed between clusters. It is not an hybrid protocol like ours. Our protocol is more relaxed, evoluting to independent checkpointing if there is no inter-cluster message.

\section{Conclusion and Future Work}

This paper describes an hybrid protocol combining coordinated and communication-induced checkpointing methods. This new approach works well with code coupling applications. It can be tuned according to the network and the application communication patterns. This protocol needs some improvements. Adding some transitivity in the dependency tracking mechanism by sending the whole $D D V$ instead of the $S N$ should allow to take less forced checkpoints. Thus more communication patterns would be efficiently supported. The protocol should tolerate multiple faults in a cluster, this implies more redundancy in the stable storage implementation. It should tolerate simultaneous fault in different clusters (the garbage collector should take care of this). The garbage collector could be more distributed. Finally, we need to implement the protocol on a real system and experiment with real applications to validate it.

\section{References}

[1] A. Agbaria and R. Friedman. Starfi sh: Fault-Tolerant Dynamic MPI Programs on Clusters of Workstations. The Eighth IEEE International Symposium on High Performance Distributed Computing, pages 167-176, August 1999.

[2] R. Badrinath and C. Morin. Common mechanisms for supporting fault tolerance in DSM and message passing systems. Technical report, July 2003.

[3] G. Bosilca, A. Bouteiller, F. Cappello, S. Djailali, G. Fedak, C. Germain, T. Herault, P. Lemarinier, O. Lodygensky, F. Magniette, V. Neri, and A. Selikhov. MPICH-V: Toward a Scalable Fault Tolerant MPI for Volatile Nodes. In Proceedings of the IEEE/ACM SC2002 Conference, pages 2947, Baltimore, Maryland, November 2002.

[4] K. Chandy and L. Lamport. Distributed Snapshots: Determining Global States of Distributed Systems. ACM Trans. Computer Systems, 3(1):63-75, February 1985.

[5] M. Costa, P. Guedes, M. Sequeira, N. Neves, and M. Castro. Lightweight Logging for Lazy Release Consistent Distributed Shared Memory. In Operating Systems Design and Implementation, pages 59-73, October 1996.

[6] M. Elnozahy, L. Alvisi, Y.-M. Wang, and D. Johnson. A Survey of Rollback-Recovery Protocols in Message-Passing Systems. ACM Computing Surveys (CSUR), 34:375-408, September 2002.

[7] S. Monnet. Conception et évaluation d'un protocole de reprise d'applications parallèles dans une fédération de grappes de calculateurs. Rapport de stage de dea, IFSIC, Université de Rennes 1, France, June 2003. In French.

[8] C. Morin, A.-M. Kermarrec, M. Banâtre, and A. Gefflaut. An Effi cient and Scalable Approach for Implementing Fault Tolerant DSM Architectures. IEEE Transactions on Computers, 49(5):414-430, May 2000.

[9] A. Nguyen-Tuong. Integrating Fault-Tolerance Techniques in Grid Applications. PhD thesis, Faculty of the School of Engineering and Applied Science at the University of Virginia, August 2000.

[10] H. Paul, A. Gupta, and R. Badrinath. Hierarchical Coordinated Checkpointing Protocol. In International Conference on Parallel and Distributed Computing Systems, pages 240 245, Novenber 2002.

[11] J. Rough and A. Goscinski. Exploiting Operating System Services to Effi ciently Checkpoint Parallel Applications in GENESIS. Proceedings of the 5th IEEE International Conference on Algorithms and Architectures for Parallel Processing, October 2002.

[12] C++SIM. http://cxxsim.ncl.ac.uk. 